Conclusion The estimation of post-operative mortality risk is a crucial part of management of patients with NSCLC. Overall mortality following surgery for NSCLC in England is currently $3 \%$ at 30 -days and $6 \%$ at 90 -days. We present UK data, stratified by age and performance status, which could be used in clinical practise to assist with the estimation of early post-operative mortality risk.

Abstract P202 Table 1 Proportion of patients who died within 90 days of lobectomy or pneumonectomy for NSCLC (italics show total number of patients who underwent the procedure in each category; \# no deaths occurred in these groups)

\begin{tabular}{cccccccc}
\hline \multicolumn{7}{c}{ Performance status } \\
\hline Age & $\mathbf{0}$ & $\mathbf{1}$ & $\mathbf{2}$ & $\mathbf{3 - 4}$ & $\mathbf{0}$ & $\mathbf{1}$ & $\mathbf{2}$ \\
\hline$<70$ & $1 \%$ & $4 \%$ & $7 \%$ & $10 \%$ & $8 \%$ & $12 \%$ & $6 \%$ \\
& 1,611 & 974 & 160 & 30 & 307 & 205 & 31 \\
$70-80$ & $4 \%$ & $7 \%$ & $9 \%$ & $13 \%$ & $19 \%$ & $14 \%$ & $22 \%$ \\
& 831 & 833 & 128 & 30 & 106 & 94 & 18 \\
$>80$ & $7 \%$ & $6 \%$ & $24 \%$ & $\#$ & $22 \%$ & $19 \%$ & $\#$ \\
& 151 & 209 & 29 & 4 & 9 & 16 & 0 \\
& & LOBECTOMY & & \multicolumn{5}{c}{ PNEUMONECTOMY } \\
\hline
\end{tabular}

\section{P203 ADDRESSING LOW SURGICAL RESECTION RATES FOR NON SMALL CELL LUNG CANCER: LOCAL INTERVENTION TO ADDRESS HIGH LEVELS OF EMERGENCY PRESENTATIONS AT LATE STAGE SHOULD BE EFFECTIVE.}

doi:10.1136/thoraxinl-2012-202678.264

EM Hadley. Barking, Havering \& Redbridge NHS Trust, London, UK

Introduction The National Lung Cancer Audit is a benchmarking tool for cancer networks and individual MDT's. The national average resection rate for all lung cancer patients is $13.7 \%$. The NLCA recommend that all MDTs with a resection rate for NSCLC below $14 \%$, and for stage $1 \mathrm{a}-2 \mathrm{~b}$ below $52 \%$ should be reviewed to ensure that patients are being offered appropriate access to thoracic surgical expertise.

In the 2011 report our MDT had a resection rate for NSCLC of $7.8 \%$. In light of this a review of all cancer patients was carried out. Results and discussion The resection rate for stage $1 \mathrm{a}-2 \mathrm{~b}$ NSCLC patients was $48 \%$ which is similar to the national average. However the number of patients presenting with operable stage $1 \mathrm{a}-2 \mathrm{~b}$ disease was unusually low at $12 \%$.

An in depth review of 18 patients with operable NSCLC showed that there were four patients that did not have a curative resection. Two were performance stage 3-4 at presentation, one had a tumour in an inoperable location and the fourth had significant comorbidities precluding surgery.

The opportunity for patients to be offered surgical treatment of stage $1 \mathrm{a}-2 \mathrm{~b}$ NSCLC at this MDT is within national targets. However the low figure on the LUCADA data is due to an unusually high proportion of patients (88\%) presenting with stage 3-4 disease. This is refected locally where we also have very high rates of lung cancer presenting via emergency admissions, around $70 \%$. The national average is around $30-40 \%$, a figure which is already considered unacceptable.

Conclusion In order to improve the rate of curative resection and improves survival for our population the key intervention is to diagnose more NSCLC at an early stage. We need to target the local population to present early, and empower primary care to refer early. We will study the impact of the 2012 National Lung Cancer Awareness Programme with interest, although it is likely that a further local campaign targeted at our specific community may be needed if we are to turn around local resection rates.

\section{P204 FRACTIONATED CARBOPLATIN AND VINOREBINE FOR ELDERLY AND POOR PERFORMANCE STATUS PATIENTS WITH NSCLC}

doi:10.1136/thoraxjnl-2012-202678.265

J Maguire, S Datta, V Kelly, P Mbanu. Liverpool Heart and Chest Hospital, Liverpool, UK

Patients with advanced NSCLC who are elderly and/or have poor Performance Status are generally considered unsuitable for chemotherapy and usually excluded from the possible benefits of systemic treatment. The outlook for this group of patients is poor, with typical survival times of four months or less.

If a tolerable form of systemic chemotherapy could be identified for elderly and poor PS patients, this would enable consideration of active rather than supportive care, and might improve overall survival rates.

We have treated 110 patients who were either aged over 70; $(16 \%, n=18)$, PS 2 or greater $(36 \%, n=40)$ or both; $(46 \%, n=52)$ with systemic chemotherapy using fractionated Carboplatin and Vinorelbine. Carboplatin was administered at AUC 2.5 on day 1 and day 8 , with oral Vinorelbine $60 \mathrm{mg} / \mathrm{m} 2$ on a 21 day cycle. 40 patients had Stage III and 68 had Stage IV disease. Prophylactic levofloxacin $500 \mathrm{mg}$ daily was given from day 9 to 20 of each treatment cycle. Renal function for Carboplatin dosage was measured using EDTA clearance. 67 patients were aged 70 or greater, and 22 more than 80 years. 90 patients were PS 2 or 3 and 51 patients were both over 70 and had poor PS

The average number of cycles given to each patient was 3. $7 \%$ of patients died within 30 days of receiving chemotherapy treatment. The median survival for the whole patient group was 7.5 months. $72 \%$ percent of patients lived for 6 months or longer from commencement of chemotherapy. $40 \%$ of patients lived 9 months or longer and $22 \%$ of patients lived for a year or longer after starting chemotherapy. There was a strong statistical correlation between response to chemotherapy and survival.

In conclusion, systemic chemotherapy using fractionated Carboplatin and Vinorelbine should be considered as a possible treatment option for patients with advanced NSCLC who are elderly or have moderate to poor PS.

\section{P205 SMOKING PREVALENCE AND SMOKING CESSATION AMONGST ACUTE MEDICAL ADMISSIONS}

doi:10.1136/thoraxjnl-2012-202678.266

R Harrison, P Preston, C Bucur, SV Fletcher. University Hospital Southampton NHS Foundation Trust, Southampton, UK

Introduction and Objectives In 2009/10 there were 1.5 million hospital admissions in adults aged $>35 y$ rs with a smoking related illness. Smoking cessation is a cost effective clinical intervention, producing better health outcomes for patients and saving NHS resources. In 2010/11 total expenditure on 'NHS Stop Smoking Services' in England excluding prescriptions was £84.3million. NICE guidelines recommend that every smoker should be offered smoking cessation advice and prescription of nicotine replacement therapy (NRT).

The purpose of this study was to identify smoking prevalence in a general medical inpatient population at the time of admission and evaluating the hospital's provision of smoking cessation advice and NRT prescription.

Methods Using a cross-sectional study design, contemporaneous data was collected from the Acute Medical Unit (AMU) on 6 separate days. All admitted patients were eligible and interviewed using a standardised questionnaire. Three interviewers agreed a standard approach to questioning. Contemporaneous hospital notes and drug charts were reviewed. Exclusions to the study included cognitive impairment, language, not being present on the ward, being significantly clinically unwell and patient refusal. 
Results Figure 1.185 patients were reviewed and 23 excluded. $85 \%$ of patients had their smoking status clearly documented. Only $30 \%$ of smokers were provided with cessation advice and 7\% were referred to Quitters. 69.5\% of smokers did not have NRT prescribed but only $19.5 \%$ not wish to receive NRT.

Conclusions The medical impatient population has a higher prevalence of smokers (28\%) when compared to the national average of $21 \%$. Our results for smoking cessation service provision compare poorly with NICE guidelines which target $100 \%$ of smokers receiving smoking cessation guidance.

Awareness needs to be raised amongst the medical staff in order to optimise the provision of advice, referral to quitters and NRT prescription rate in order to achieve the targets set by NICE and the $\mathrm{DOH}$. We are currently embarking upon a series of educational sessions and a ward round 'checklist' is under development which will include a prompt for smoking cessation.

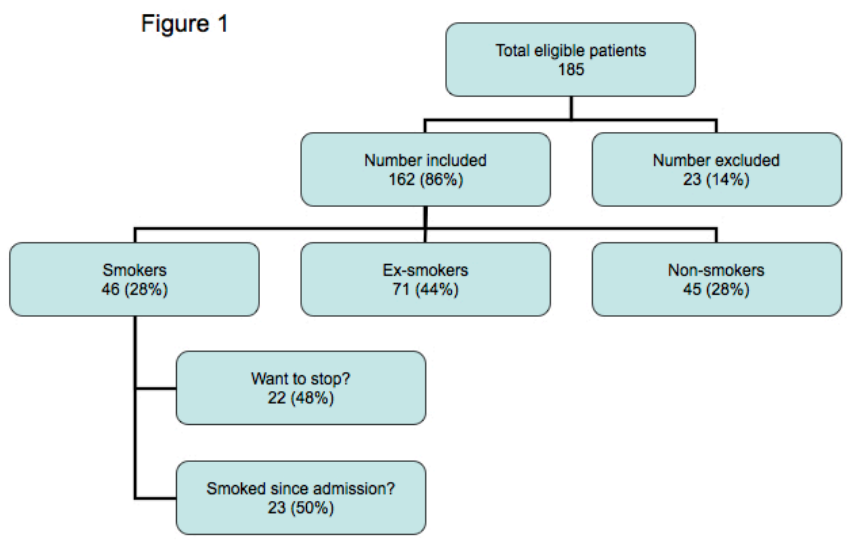

Abstract P205 Figure 1

\section{Clinical management of patients with COPD}

\section{P206 THE ACCEPTABILITY OF STARTING NON-INVASIVE VENTILATION AT HOME TO PATIENTS WITH COPD AND THEIR CARERS}

doi:10.1136/thoraxjnl-2012-202678.267

M Jawad, NK Gale, AM Turner. University of Birmingham, Birmingham, United Kingdom

Introduction Home non-invasive ventilation (NIV) is prescribed to patients suffering from severe chronic obstructive pulmonary disease (COPD) with chronic respiratory failure. Uncertainty exists regarding clinical efficacy and the effect on a patient's quality of life (OOL) during long term use. This qualitative study aimed to explore the perceptions and experiences of healthcare professionals (HCPs) involved in the management of COPD patients, COPD patients themselves and the patient's carers regarding the use of home NIV, with the aim of understanding decision making processes and improving its future use.

Method 15 HCPs including doctors, nurses, physiotherapists and physiologists, 20 COPD patients with moderate to very severe disease and 4 carers ( 3 patient spouses and 1 patient sibling) were recruited to participate in a semi-structured interview from a tertiary NIV hospital, a hospital providing home NIV services and the community. All interviews were transcribed verbatim and analysed using framework analysis.

Results Two major themes emerged from the interviews. The need for 'adapting to home NIV' was reported by all patients. This corresponds to the need for acceptance of the NIV and the ability to overcome NIV specific problems. Patients expressed that this change was forced upon them by their HCPs. It was evident from the interviews that carers also experienced this need for adaptation. Another theme that emerged from the interviews was of 'negotiating the evidence'. HCPs reported that due to the lack of evidence and guidance available for home NIV use, they encountered difficulties when deciding which patients to start or continue on home NIV. Patients faced a dilemma of whether to accept the doctor's 'orders' to use home NIV, despite distressing NIV experiences at hospital. Nevertheless improvements in the patient's QOL, particularly in their day-to-day functions, were reported.

Conclusion This study demonstrated that patients find the use of home NIV acceptable once they have adapted to it. Recommendations include providing patients with greater technical NIV support, a better experience of NIV at hospital and improving communication between HCPs and patients to allow for greater understanding of the patient's perspective.

\section{P207 CAN HOLISTIC INTERVENTIONS IMPROVE THE CARE OF PEOPLE WITH SEVERE CHRONIC OBSTRUCTIVE PULIMONARY DISEASE (COPD)? A SYSTEMATIC REVIEW}

doi:10.1136/thoraxjnl-2012-202678.268

UB Nurmatov, S Buckingham, M Kendall, A Sheikh, H Pinnock. The University of Edinburgh, Edinburgh, Scotland, UK

Introduction and Objectives People with severe COPD have a long-recognised burden of disabling physical symptoms compounded by co-morbidity, psychological distress and social isolation. We aimed to review the effectiveness of interventions designed to deliver holistic care compared to usual care for people with severe COPD.

Methods We searched 11 international electronic databases, three trial repositories and contacted a panel of international experts to locate published, unpublished and in-progress randomised controlled trials (RCTs), quasi-RCTs and controlled clinical trials (CCTs) that investigated holistic interventions designed to support patients with severe COPD in any healthcare context. Date range January 1990-March 2012; no language or geographical restrictions. Quality assessment and data extraction followed the Cochrane Collaboration method. Health-related quality of life (HROoL) was the primary outcome. We used a piloted data extraction sheet and undertook narrative synthesis.

Results From 2,866 potentially relevant papers, three trials met our inclusion criteria: two RCTs (from United States and Australia), and one CCT (from Thailand) studying a total of 216 patients. Critical appraisal identified a moderate (one RCT and the CCT) or high (one RCT) risk of bias. All interventions were led by nurses acting in a co-ordinating role (e.g. facilitating community support in Thailand, providing case-management in the US, or co-ordinating inpatient care in Australia). The community-based intervention in Thailand significantly improved HRQoL at three months compared to (limited) usual care (St George's Respiratory Questionnaire: intervention: $30.3 \pm 19.4$ vs control $52.4 \pm 21.3 p<0.001)$. Significant effects in the US trial were confined to 'Physical functioning' and 'General health' sub-domains of Medical Outcomes Study ShortForm-36 at three but not six-months. There were no significant changes in the Australian trial.

Conclusions Some 15 years after reports first highlighted the substantial unmet needs of people with severe COPD, we have been unable to find robust trial evidence about interventions to address those needs. There is an urgent need to develop and evaluate interventions to deliver or enhance holistic care and improve the quality of life of people with severe COPD

Funding Dunhill Medical Trust. HP is supported by a PCRCA Fellowship (CSO, Scottish Government). 